\title{
Disposition Kinetics of Levofloxacin in Sheep after Intravenous and Intramuscular Administration
}

\author{
Ayman Goudah $^{1}$ and Sherifa Hasabelnaby ${ }^{2}$ \\ ${ }^{1}$ Pharmacology Department, Faculty of Veterinary Medicine, Cairo University, Giza, P.O. Box 12211, Egypt \\ ${ }^{2}$ College of Pharmacy, Ohio State University, Columbus, OH 43210, USA
}

Correspondence should be addressed to Ayman Goudah, aymangouda@yahoo.com

Received 31 May 2010; Revised 29 July 2010; Accepted 5 October 2010

Academic Editor: William Ravis

Copyright (C) 2010 A. Goudah and S. Hasabelnaby. This is an open access article distributed under the Creative Commons Attribution License, which permits unrestricted use, distribution, and reproduction in any medium, provided the original work is properly cited.

The present study was planned to investigate the disposition kinetics of levofloxacin in plasma of female native Barky breed sheep after single intravenous (IV) and intramuscular (IM) administration of $4 \mathrm{mg} / \mathrm{kg}$ body weight. The concentrations of levofloxacin in the plasma were measured using high-performance liquid chromatography (HPLC) with a UV detector on samples collected at 0 , $0.08,0.16,0.33,0.5,1,2,4,6,8,10,12,18,24,32$, and $48 \mathrm{~h}$ after treatment. Following intravenous injection, the decline in plasma drug concentration was biexponential with half-lives of $\left(t_{1 / 2 \alpha}\right) 0.33 \pm 0.12 \mathrm{~h}$ and $\left(t_{1 / 2 \beta}\right) 3.29 \pm 0.23 \mathrm{~h}$ for distribution and elimination phases, respectively. The volume of distribution at steady state $V_{(\mathrm{d}(\mathrm{ss}))}$ was $0.86 \pm 0.23 \mathrm{l} / \mathrm{kg}$. After intramuscular administration of levofloxacin at the same dose, the peak plasma concentration $\left(C_{\max }\right)$ was $3.1 \pm 0.35 \mu \mathrm{g} / \mathrm{mL}$ and was obtained at $1.64 \pm 0.29 \mathrm{~h}$ $\left(T_{\max }\right)$, the elimination half-life $\left(T_{1 / 2 \mathrm{el}}\right)$ was $3.58 \pm 0.30 \mathrm{~h}$, and AUC was $20.24 \pm 1.31 \mu \mathrm{g} . \mathrm{h} / \mathrm{mL}$. The systemic bioavailability was $91.35 \pm 6.81 \%$. In vitro plasma protein binding was $23.74 \%$. When approved therapy fails, levofloxacin may be used in some countries for therapy of food animals, however, that is not true in the US.

\section{Introduction}

Levofloxacin is a recently introduced third-generation fluoroquinolones with high activity against a wide spectrum of Gram-positive and Gram-negative bacteria [1]. In human clinical trials, levofloxacin has been found to be very effective in the treatment of infections of upper and lower respiratory tract, genitourinary system, and skin and soft tissue [2]. Compared to other fluoroquinolones, ofloxacin and ciprofloxacin, levofloxacin has more pronounced bactericidal activity against organisms such as Pseudomonas and Enterobacteriaceae [3]. The bactericidal effect of levofloxacin is achieved through reversible binding to DNA gyrase and subsequent inhibition of bacterial DNA replication and transcription [4-6]. Fluoroquinolones act by a concentrationdependent killing mechanism, whereby the optimal effect is attained by the administration of high doses over a short period of time [7]. This concentration-dependent killing profile is associated with a relatively prolonged postantibiotic effect [8]. For this class of antimicrobials, drug exposure, as measured by the area under the plasma concentration versus time curve (AUC), has been used to calculate surrogate efficacy indices, such as the AUC/MIC ratio, where MIC stands for the in vitro minimal inhibiting concentration of the tested bacteria [9-11]. Thus, variations of drug exposure can be associated with variations in the probability of a successful outcome with a specific dosage regime.

The drug undergoes a limited metabolism in rats and human [12] and is primarily excreted by kidney mainly as active drug. Inactive metabolites ( $\mathrm{N}$-oxide and desmethyl metabolites) represent $<5 \%$ of the total dose [13], as other fluoroquinolones are metabolised in chickens as reported in [14-16], as chickens metabolized marbofloxacin to Ndesmethyl-marbofloxacin.

The pharmacokinetics of levofloxacin has been investigated in a limited number of animal species including rats [17], rabbits [18], calves [19, 20], goats [21], cats [22], male camels [23], and stallions [24]. However, there is no available information on the kinetics of levofloxacin in the sheep. Therefore, the present study was undertaken to determine 
the disposition kinetics and bioavailability of levofloxacin in sheep following a single intravenous (IV) or intramuscular (IM) administration of $4 \mathrm{mg} / \mathrm{kg}$ bwt.

\section{Material and Methods}

2.1. Drugs and Chemicals. Levaquin $(25 \mathrm{mg} / \mathrm{mL}$ of levofloxacin solution) was obtained from Janssen Pharmaceutica $\mathrm{N}$ $\mathrm{V}$ (Beerse, Belgium). Ciprofloxacin as internal standard was purchased from Sigma, Chemical Company (St. Louis, MO, USA). The solvents (Baker Inc., Phillipsburg, NJ, USA) used during the chromatographic analysis of the drug were HPLC grade.

2.2. Experimental Animals. The study was approved by the Animal Care and Use Committee at the Faculty of Veterinary Medicine, Cairo University. We used ten female native Barky breed 2-3 years old, 45-55 kg body weight. The animals were in optimal nutritional condition, fed on concentrated pellets, hay, and alfalfa, and had free access to water ad libitum daily. The health of all animals was monitored prior to and throughout the experimental period.

2.3. Drug Administration. The study was performed in two phases, following a crossover design $(5 \times 5)$. Animals were randomly assigned into two groups, with each group containing five animals. In phase one of the study, five animals were given a single intravenous injection into the left jugular vein at dose of $4 \mathrm{mg} / \mathrm{kg}$ bodyweight levofloxacin, and the other five were injected intramuscularly into the lower third region of the neck muscles with the drug at the same dose. Three ml venous whole blood samples were taken by jugular venepuncture into $10 \mathrm{~mL}$ heparinized Vacutainers (Becton Dickinson vacutainer Systems, Rutherford, NJ, USA). The sampling times were 0 (blank sample), 0.08, 0.16, 0.33, 0.5, $1,2,4,6,8,10,12,18,24,32$, and $48 \mathrm{~h}$ after treatment. All the blood samples were centrifuged at $3000 \mathrm{~g}$ for $15 \mathrm{~min}$ to separate the plasma. The plasma samples were frozen at $-20^{\circ} \mathrm{C}$ until analysed. After a washout period of 2 weeks, the animals that had been injected intravenously with the drug were injected intramuscularly and vice versa. Pilot studies have shown that a 2-week period is enough to avoid carry over effect. Blood was collected and processed as above. The heparinized plasma samples were frozen at $-20^{\circ} \mathrm{C}$ and analyzed by high-performance liquid chromatography (HPLC). All samples were analyzed within one week after each experimental phase.

2.4. Analytical Method. Plasma concentrations of levofloxacin were measured using a modified HPLC method [25]. Briefly, the HPLC system was performed on Shimadzu Liquid Chromatography System (Duisburg, Germany) equipped with an LC9A pump, an automatic sampler SIL6B, and a UV detector. Class LC 10 software version 1.6 (Shimadzu) was used for data analysis and processing. Levofloxacin and ciprofloxacin (as internal standard, 99.2 pure, $1 \mu \mathrm{g} 10 / \mu \mathrm{L}$ methanol) were isolated from plasma. The plasma proteins were removed via methanol precipitation; $200 \mu \mathrm{l}$ plasma were mixed with $400 \mu \mathrm{l}$ methanol and vigor- ously shaken. The precipitated proteins were removed via centrifugation at $12000 \times \mathrm{g}$ for $5 \mathrm{~min}$. Subsequently, $20 \mu \mathrm{l}$ of the supernatant were injected onto the column.

The HPLC separation was performed using a reversedphase $\mathrm{C}_{18}$ column (Discovery, Supelco, $5 \mu \mathrm{m}, 4.6 \mathrm{~mm} \times$ $150 \mathrm{~mm}$ ) with an injection volume of $20 \mu \mathrm{l}$. The mobile phase consisted of water: acetonitrile ( $80: 20, \mathrm{v} / \mathrm{v})$ with $0.3 \%$ of triethylamine and $\mathrm{pH}$ adjusted to 3.3 with phosphoric acid, using an isocratic form with a flow rate of $1.0 \mathrm{~mL} / \mathrm{min}$. The detector wavelength was set at $295 \mathrm{~nm}$. The analytes were identified from the retention times of $97-99 \%$ pure reference standards.

The calibration curves of plasma were prepared with seven different concentrations between 0.01 and $10 \mu \mathrm{g} / \mathrm{mL}$ using blank sheep plasma. A calibration curve was obtained by plotting the peak height ratio versus the nominal concentrations. The equation was calculated by the least-squares method using linear regression. The limit of quantification (LOQ) based on a signal-to-noise ratio $>5$ was $0.04 \mu \mathrm{g} / \mathrm{mL}$ of levofloxacin in supplemented sheep plasma. Under our experimental conditions, the linearity of the method was from 0.01 to $10 \mu \mathrm{g} / \mathrm{mL}$ of levofloxacin sheep plasma, and the value of correlation coefficients $(r)$ was $>0.99$. The peak height ratios of an unknown specimen (peak height of levofloxacin/peak height of internal standard) were compared with that of the standard.

The precision and accuracy of the method were evaluated by repetitive analysis of the plasma samples $(n=12)$ spiked with different known concentrations of levofloxacin. The percentage recoveries were determined by comparing the peak height of blank samples spiked with different amounts of drug and treated as any sample, with the peak height of the same standards prepared in phosphate buffer $(n=$ $6)$. Intra-assay variations were determined by measuring six replicates $(n=6)$ of three standard samples used for calibration curves. The intra-assay variation coefficients were $<4.3 \%$. Interassay precisions were determined by assaying the three standard samples on three separate days. The Interassay variation coefficients were $<4.6 \%$. Recovery of levofloxacin from plasma was found to be $93 \%$.

2.5. In Vitro Plasma Protein Binding. The extent of plasma protein binding was determined in vitro using ultrafiltration [26]; antimicrobial-free plasma from sheep fortified with known concentrations of levofloxacin $(0.01,0.16,0.32$, $1.25,5$, and $10 \mu \mathrm{g} / \mathrm{mL}$ ) was used. One $\mathrm{ml}$ of each sample was placed on a conditioned semipermeable membrane (Centriflow Cones CF-50, Amicon Corp., Lexington, MA, USA) resting on porous conical polyethylene support on the top of centrifuge tubes. The tubes were centrifuged at $1500 \mathrm{~g}$ for 45-60 min. Plasma samples and their corresponding ultrafiltrates were assayed by the same method (HPLC) as described above. The percentage of plasma protein binding was calculated according to the following equation:

Protein binding \%

$=\frac{\text { Total concentration }- \text { Ultrafiltrate concentration }}{\text { Total concentration }} \times 100$. 


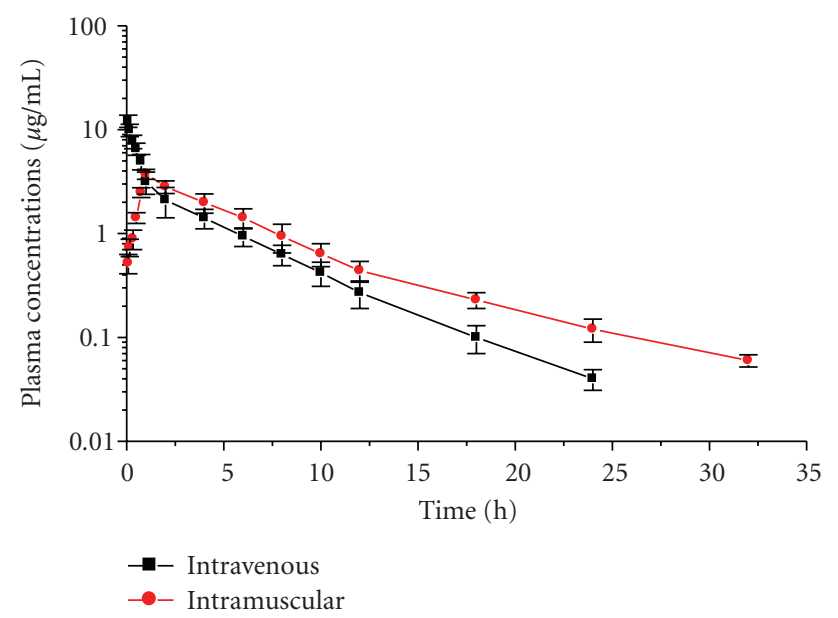

Figure 1: Mean \pm SD plasma concentrations of levofloxacin in sheep after intravenous $(\boldsymbol{\square})$ and intramuscular $(\bullet)$ injection of $4 \mathrm{mg} / \mathrm{kg}$ b.wt. $(n=10)$.

2.6. Pharmacokinetic Analysis. Pharmacokinetic analysis of plasma levofloxacin concentration versus time data was conducted by noncompartmental analysis using WinNonLin Professional version 4.1 software package (Pharsight Corporation, Mountain View, California). For the intravenous data, the appropriate pharmacokinetic model was determined by visual examination of individual concentrationtime curves and by application of Akaike's Information Criterion (AIC) [27]. The plasma concentration-time relationship was best estimated as a two-compartment open model:

$$
C_{p}=A e^{-\alpha t}+B e^{-\beta t},
$$

where $C_{p}$ is the concentration of drug in the plasma at time $t$, $A$ and $B$ are the zero-time drug intercepts of the distribution and elimination phase expressed as $\mu \mathrm{g} / \mathrm{mL}, \alpha$ and $\beta$ are the distribution and elimination rate constants expressed in units of reciprocal time $\left(h^{-1}\right)$, and $e$ is the natural logarithm base. The distribution and elimination half-lives $\left(t_{1 / 2 \alpha}\right.$ and $\left.t_{1 / 2 \beta}\right)$ were calculated according to standard equations [28], while the volume of distribution at steady state $\left(V_{(\mathrm{d}(\mathrm{ss}))}\right)$ and the mean residence time (MRT) were calculated according to the following equations $\left(V_{(\mathrm{d}(\mathrm{ss}))}=\mathrm{Cl}_{B} \times \mathrm{MRT}\right.$ and $\mathrm{MRT}=$ AUMC/AUC), respectively.

Following IM administration of levofloxacin, plasma concentrations data were analyzed by both compartmental and noncompartmental methods based on the statistical moment theory [28]. The terminal elimination half-life $\left(t_{1 / 2 \mathrm{el}}\right)$ and absorption half-life $\left(t_{1 / 2(a)}\right)$ were calculated as $\ln 2 / k_{\mathrm{el}}$ or $\ln 2 / k_{\mathrm{a}}$, respectively, where $k_{\mathrm{el}}$ and $k_{\mathrm{ab}}$ are the elimination and absorption rate constant, respectively. The areas under the concentration-time curves (AUC) were calculated by the trapezoidal rule and further extrapolated to infinity by dividing the last experimental plasma concentration by the terminal slope $(\beta)$. The mean residence time (MRT) was calculated as AUMC/AUC, where AUMC is the area under the first moment curve, each individual curve of levofloxacin over time was analyzed to determine the peak concentration
$C_{\max }$ (extrapolated from the curve), and the time to peak concentration $T_{\max }$ was read from the data. The systemic clearance was calculated as $\mathrm{Cl}=$ Dose/AUC. The absolute bioavailability $(\mathrm{F} \%)$ was calculated as $\left(\mathrm{AUC}_{\mathrm{IM}} / \mathrm{AUC}_{\mathrm{IV}}\right) \times$ 100. In case of extravascular administration, the volume of distribution at steady state $\left(V_{(\mathrm{d}(\mathrm{ss}))}\right)$ and the systemic clearance $\left(\mathrm{Cl}_{B}\right)$ were calculated according to the following equations $\left(V_{(\mathrm{d}(\mathrm{ss}))}=\mathrm{Vd} / \mathrm{F}\right)$ and $\mathrm{Cl}_{B}=\mathrm{Cl}_{B} / \mathrm{F}$, respectively.

Pharmacodynamic efficacy of levofloxacin was determined by calculating the $C_{\max } / \mathrm{MIC}$ and $\mathrm{AUC}_{24} / \mathrm{MIC}$ ratios following IM administrations using the respective mean MIC value for susceptible Klebsiella spp. $(0.06 \mu \mathrm{g} / \mathrm{mL})$, Shigella spp. $(0.06 \mu \mathrm{g} / \mathrm{mL})$, Salmonella spp. $(0.12 \mu \mathrm{g} / \mathrm{mL})$, Proteus spp. $(0.06 \mu \mathrm{g} / \mathrm{mL})$, and Acinetobacter spp. $(0.12 \mu \mathrm{g} / \mathrm{mL})$ according to Marshall and Jones [29]; these values were derived from those determined in the studies involving antibacterial activity of levofloxacin against strains isolated from human beings.

2.7. Statistical Analysis. The statistical analysis was performed using the SPSS 17.1 software package (SAS, Cary, NC, USA). Results are presented as arithmetic mean \pm SD. The nonparametric Wilcoxon test was used to compare the parameters obtained after intravenous and intramuscular administration. Means were considered significantly different at $P<.05$.

\section{Results}

Clinical examination of all animals before and after each trial did not reveal any abnormalities. No adverse reactions were observed after the single-dose IV or IM administration of levofloxacin in the animals studied. Akaike's Information Criterion test indicated that a two-compartment model best represented the plasma concentration versus time data after IV administration of levofloxacin in sheep.

The mean plasma concentration-time profiles of levofloxacin following single IV and IM administrations of $4 \mathrm{mg} / \mathrm{kg}$ b.wt are presented graphically in Figure 1. Mean $\pm \mathrm{SD}$ values of pharmacokinetic parameters estimated from the curve fitting are shown in Table 1 . In vitro plasma protein binding of levofloxacin was $23.74 \%$. Following intramuscular administrations of levofloxacin using $\mathrm{MIC} \leq$ $0.12 \mu \mathrm{g} / \mathrm{mL}$, the $C_{\max } / \mathrm{MIC}_{90}$ ratio was 25.83 -fold, and the $\mathrm{AUC}_{0-24} / \mathrm{MIC}_{90}$ ratio was $160.42 \mathrm{~h}$.

\section{Discussion}

Plasma levofloxacin disposition curves after IV injection were best fit to an open bicompartmental model in all the animals, which is in accordance with the results reported for calves and lactating goats, respectively, [20, 21].

The $V_{(\mathrm{d}(\mathrm{ss}))}$ is a clearance-independent volume of distribution that is used to calculate the drug amount in the body under equilibrium conditions [30]. Fluoroquinolones are lipid-soluble drugs that have a large volume of distribution [31]. The $\left.V_{(\mathrm{d}(\mathrm{ss})}\right)$ for levofloxacin was $0.861 / \mathrm{kg}$ in sheep indicating a relatively wide distribution after 
TABLE 1: Mean $\pm S D$ plasma pharmacokinetic parameters of levofloxacin $(\mu \mathrm{g} / \mathrm{mL})$ in sheep $(n=10)$ following IV and IM administration at a dose rate of $4 \mathrm{mg} / \mathrm{kg}$ b.w.

\begin{tabular}{|c|c|c|c|}
\hline Parameters & Unit & IV & IM \\
\hline$\alpha$ & $\mathrm{h}^{-1}$ & $2.19 \pm 0.17$ & - \\
\hline$k_{\mathrm{a}}$ & $\mathrm{h}^{-1}$ & - & $1.39 \pm 0.15$ \\
\hline$t_{1 / 2 \alpha}$ & $\mathrm{h}$ & $0.33 \pm 0.12$ & - \\
\hline$t_{1 / 2(a)}$ & $\mathrm{h}$ & - & $0.51 \pm 0.11$ \\
\hline$\beta$ & $\mathrm{h}^{-1}$ & $0.19 \pm 0.09$ & - \\
\hline$k_{\mathrm{el}}$ & $\mathrm{h}^{-1}$ & - & $0.21 \pm 0.04$ \\
\hline$t_{1 / 2 \beta}$ & $\mathrm{h}$ & $3.29 \pm 0.23$ & - \\
\hline$t_{1 / 2 \mathrm{el}}$ & $\mathrm{h}$ & - & $3.58 \pm 0.30$ \\
\hline$V_{(\mathrm{d}(\mathrm{ss}))}$ & $1 / \mathrm{kg}$ & $0.86 \pm 0.23$ & $1.02 \pm 0.18$ \\
\hline $\mathrm{Cl}_{B}$ & L/h.kg & $0.20 \pm 0.05$ & $0.19 \pm 0.03$ \\
\hline AUC & $\mu \mathrm{g} \cdot \mathrm{h} / \mathrm{mL}$ & $21.61 \pm 1.24$ & $20.24 \pm 1.31^{*}$ \\
\hline MRT & $\mathrm{h}$ & $4.26 \pm 0.94$ & $5.33 \pm 1.05^{*}$ \\
\hline$C_{\max }$ & $\mu \mathrm{g} / \mathrm{mL}$ & $12.17 \pm 1.73$ & $3.10 \pm 0.35$ \\
\hline$T_{\max }$ & $\mathrm{h}$ & - & $1.64 \pm 0.29$ \\
\hline$F$ & $\%$ & - & $91.35 \pm 6.81$ \\
\hline
\end{tabular}

$\beta\left(k_{\mathrm{el}}\right)$ : elimination rate constant; $\alpha\left(k_{a}\right)$ : distribution (absorption) rate constant; $t_{1 / 2 \alpha}$ : distribution half-life; $t_{1 / 2(\mathrm{a})}$ : absorption half-life; $t_{1 / 2 \beta}\left(t_{1 / 2 \mathrm{el}}\right)$ : elimination half-life; $V_{(\mathrm{d}(\mathrm{ss}))}$ : volume of distribution; $\mathrm{Cl}_{B}$ : total body clearance; AUC: area under the curve from zero to infinity by the trapezoidal integral; MRT: mean residence time; $C_{\max }$ : maximum plasma concentration; $T_{\max }$ : time to peak concentration; $\mathrm{F}(\%)$ : bioavailability; for $\mathrm{IM},\left(V_{(\mathrm{d}(\mathrm{ss}))}=V d / F\right)$ and $\mathrm{Cl}_{B}=\mathrm{Cl}_{B} / F$

${ }^{*} P<.05$.

IV administration and it was slightly differing from that reported for levofloxacin in lactating goats $0.731 / \mathrm{kg}[21]$ and moxifloxacin in lactating goats $(0.791 / \mathrm{kg})$ [32]. The discrepancies between values calculated for pharmacokinetic parameters may be attributed to the animal species, the drug formulation employed, the age, size or sex of the animals, to differences in fatty tissue deposits between animal species or breeds, or even to interindividual variations and also due to the method of analysis of the drug [33].

The clearance of levofloxacin in sheep was $0.2 \mathrm{~L} / \mathrm{h} . \mathrm{kg}$ similar to those values reported in calves and lactating goats 0.19 and $0.18 \mathrm{~L} / \mathrm{h} . \mathrm{kg}[20,21]$, respectively.

The elimination half-life of levofloxacin following IV administration was $3.29 \mathrm{~h}$. This value is close to that reported for levofloxacin in lactating goats $2.95 \mathrm{~h} \mathrm{[21]} \mathrm{and} \mathrm{longer} \mathrm{than}$ that reported in calves [20] $1.61 \mathrm{~h}$, and shorter than that reported in rabbits $7.50 \mathrm{~h} \mathrm{[18].}$

Following intramuscular injection, the present data were best represented by a one-compartment model and the estimated $C_{\max }(3.10 \mu \mathrm{g} / \mathrm{mL})$ was similar to that data reported in calves and lactating goats $(3.07$ and $3.16 \mu \mathrm{g} / \mathrm{mL})$ $[19,21]$, respectively.

The time to reach the maximum concentration of levofloxacin in sheep $\left(T_{\max }=1.64 \mathrm{~h}\right)$ was longer than that recorded levofloxacin in calves $(1.00 \mathrm{~h})$ [19] and shorter than that reported in lactating goats $1.76 \mathrm{~h}$ [21]. The absorption process of levofloxacin was moderately fast as showed by the absorption rate constant $\left(k_{a}\right) 1.39 \mathrm{~h}^{-1}$, short absorption half-life $\left(\mathrm{t}_{1 / 2(a)}\right) 0.51 \mathrm{~h}$, and confirmed by the short $T_{\max }(1.64 \mathrm{~h})$. The overall MRT was longer for IM administration compared with that for IV injection, with an estimated time of $5.33 \mathrm{~h}$. This was expected as the MRT after IM injection depends on both the disposition and absorption rates. The MRT of levofloxacin was similar to that recorded for calves and lactating goats $(5.57$ and $5.24 \mathrm{~h})[19,21]$, respectively.

The elimination half-life value was $3.58 \mathrm{~h}$ after IM administration, similar to that recorded for calves and lactating goats (3.67 and $3.64 \mathrm{~h}$ ) $[19,21]$, respectively.

The systemic bioavailability of levofloxacin in sheep after IM administration was $91.35 \%$, and the absorption process was rapid with absorption half-life $\left(t_{1 / 2(a)}\right) 0.51 \mathrm{~h}$. This value indicates the excellent absorption of the drug from that injection site. This value was similar to values reported for levofloxacin in lactating goats [21] and moxifloxacin in sheep [34] and that reported value was effectively higher than that reported for calves $56.6 \%$ [19].

Protein binding has long been considered one of the most important physicochemical characteristics of drugs, playing a potential role in distribution, excretion, and therapeutic effectiveness as a low protein binding generally enables a rapid and extensive distribution into the intracellular and extracellular space [35]. In this study, the in vitro plasma protein binding experiment showed that levofloxacin displayed a low level of binding to plasma proteins (approximately $23.74 \%$ ) to sheep plasma. The results of in vitro protein binding may differ substantially depending on the methodology and experimental conditions [36]. The low 
protein binding of levofloxacin in sheep plasma proteins is in agreement with previously reported value of $22 \%$ in lactating goats [21], 24\% in human [12] and 25\% in rabbits [18]. Nevertheless, it was relatively lower to that reported $(17 \%)$ in calves [19].

It has been established that for concentration-dependant fluoroquinolones, the $\mathrm{AUC}_{0-24} / \mathrm{MIC}_{90}$ ratio is the most important efficacy predictor, with the rate of clinical cure being greater than $80 \%$ when this ratio is higher than 100-125 [37]. A second predictor of efficacy for concentration dependent antibiotic is the ratio $C_{\max } / \mathrm{MIC}_{90}$, considering that values $\geq 10$ would lead to better clinical results [38]. High $C_{\max } / \mathrm{MIC}_{90}$ ratios have been associated with a lower incidence resistance development [39]. It is suggested that the critical break points determining the efficacy of fluoroquinolones are $C_{\max } / \mathrm{MIC}_{90} \geq 8-10$, and $\mathrm{AUC}_{0-24} / \mathrm{MIC}_{90} \geq 100[38,39]$. The MIC of levofloxacin has not yet been determined for bacteria isolated from sheep. To cover most of the susceptible organisms, in this discussion, the $\mathrm{MIC}_{90}$ of $0.12 \mu \mathrm{g} / \mathrm{mL}$ of levofloxacin has been taken into consideration [29]. Based on this data, a dosage of $4 \mathrm{mg} / \mathrm{kg}$ levofloxacin IM in sheep would result in a $C_{\max } / \mathrm{MIC}_{90}$ ratio of 25.83-fold, which exceeds the recommended ratio of 10 . The second surrogate marker $\mathrm{AUC}_{24} / \mathrm{MIC}_{90}$ was $160.42 \mathrm{~h}$. Based on the calculated $C_{\max } / \mathrm{MIC}_{90}$ and $\mathrm{AUC}_{0-24} / \mathrm{MIC}_{90}$, a dosage of $4 \mathrm{mg} / \mathrm{kg}$ b.wt. is recommended to treat infections caused by bacteria with $\mathrm{MIC} \leq 0.12 \mu \mathrm{g} / \mathrm{mL}$.

It can be concluded that levofloxacin administered intravenously or intramuscularly in the applied dosing schedule is efficacious against bacteria with $\mathrm{MIC} \leq 0.12 \mu \mathrm{g} / \mathrm{mL}$. Consequently, levofloxacin could be useful in the treatment of systemic infections in sheep after specific assessment of susceptible micro-organisms, also, when approved therapy fails, levofloxacin may be used in some countries for therapy of food animals, however, that is not true in the US. Only enrofloxacin is approved for beef cattle and cannot be used extralabel.

\section{References}

[1] M. Martinez, P. McDermott, and R. Walker, "Pharmacology of the fluoroquinolones: a perspective for the use in domestic animals," Veterinary Journal, vol. 172, no. 1, pp. 10-28, 2006.

[2] R. Davis and H. M. Bryson, "Levofloxacin: a review of its antibacterial activity, pharmacokinetics and therapeutic efficacy," Drugs, vol. 47, no. 4, pp. 677-700, 1994.

[3] N. Klesel, K. H. Geweniger, P. Koletzki et al., "Chemotherapeutic activity of levofloxacin (HR 355, DR-3355) against systemic and localized infections in laboratory animals," Journal of Antimicrobial Chemotherapy, vol. 35, no. 6, pp. 805-819, 1995.

[4] J. S. Wolfson and D. C. Hooper, "Fluoroquinolone antimicrobial agents," Clinical Microbiology Reviews, vol. 2, no. 4, pp. 378-424, 1989.

[5] K. Drlica and X. Zhao, "DNA gyrase, topoisomerase IV, and the 4-quinolones," Microbiology and Molecular Biology Reviews, vol. 61, no. 3, pp. 377-392, 1997.

[6] G. A. Albarellos, L. A. Ambros, and M. F. Landoni, "Pharmacokinetics of levofloxacin after single intravenous and repeat oral administration to cats," Journal of Veterinary Pharmacology and Therapeutics, vol. 28, no. 4, pp. 363-369, 2005.
[7] G. L. Drusano, D. E. Johnson, M. Rosen, and H. C. Standiford, "Pharmacodynamics of a fluoroquinolone antimicrobial agent in a neutropenic rat model of Pseudomonas sepsis," Antimicrobial Agents and Chemotherapy, vol. 37, no. 3, pp. 483-490, 1993.

[8] F. S. Aliabadi and P. Lees, "Pharmacokinetics and pharmacodynamics of danofloxacin in serum and tissue fluids of goats following intravenous and intramuscular administration," American Journal of Veterinary Research, vol. 62, no. 12, pp. 1979-1989, 2001.

[9] J. M. Hyatt, P. S. McKinnon, G. S. Zimmer, and J. J. Schentag, "The importance of pharmacokinetic/pharmacodynamic surrogate markers to outcome: focus on antibacterial agents," Clinical Pharmacokinetics, vol. 28, no. 2, pp. 143-160, 1995.

[10] J. B. Meinen, J. T. McClure, and E. Rosin, "Pharmacokinetics of enrofloxacin in clinically normal dogs and mice and drug pharmacodynamics in neutropenic mice with Escherichia coli and staphylococcal infections," American Journal of Veterinary Research, vol. 56, no. 9, pp. 1219-1224, 1995.

[11] W. A. Craig, "Pharmacokinetic/pharmacodynamic parameters: rationale for antibacterial dosing of mice and men," Clinical Infectious Diseases, vol. 26, no. 1, pp. 1-12, 1998.

[12] H. D. Langtry and H. M. Lamb, "Levofloxacin: its use in infections of the respiratory tract, skin, soft tissues and urinary tract," Drugs, vol. 56, no. 3, pp. 487-515, 1998.

[13] M. Hurst, H. M. Lamb, L. J. Scott, and D. P. Figgitt, "Levofloxacin: an updated review of its use in the treatment of bacterial infections," Drugs, vol. 62, no. 14, pp. 2127-2167, 2002.

[14] A. Anadon, M. R. Martinez-Larranaga, M. J. Diaz et al., "Pharmacokinetics and residues of enrofloxacin in chickens," American Journal of Veterinary Research, vol. 56, no. 4, pp. 501-506, 1995.

[15] A. Anadón, M. R. Martínez-Larrañaga, M. J. Díaz et al., "Pharmacokinetic characteristics and tissue residues for marbofloxacin and its metabolite N-desmethyl-marbofloxacin in broiler chickens," American Journal of Veterinary Research, vol. 63, no. 7, pp. 927-933, 2002.

[16] A. Anadón, M. A. Martínez, M. Martínez, C. De La Cruz, M. J. Díaz, and M. R. Martínez-Larrañaga, "Oral bioavailability, tissue distribution and depletion of flumequine in the food producing animal, chicken for fattening," Food and Chemical Toxicology, vol. 46, no. 2, pp. 662-670, 2008.

[17] F. C. Cheng, T. R. Tsai, Y. F. Chen, L. C. Hung, and T. H. Tsai, "Pharmacokinetic study of levofloxacin in rat blood and bile by microdialysis and high-performance liquid chromatography," Journal of Chromatography A, vol. 961, no. 1, pp. 131-136, 2002.

[18] C. J. Destache, C. B. Pakiz, C. Larsen, H. Owens, and A. K. Dash, "Cerebrospinal fluid penetration and pharmacokinetics of levofloxacin in an experimental rabbit meningitis model," Journal of Antimicrobial Chemotherapy, vol. 47, no. 5, pp. 611$615,2001$.

[19] V. K. Dumka and A. K. Srivastava, "Pharmacokinetics, urinary excretion and dosage regimen of levofloxacin following a single intramuscular administration in cross bred calves," Journal of Veterinary Science, vol. 7, no. 4, pp. 333-337, 2006.

[20] V. K. Dumka and A. K. Srivastava, "Disposition kinetics, urinary excretion and dosage regimen of levofloxacin formulation following single intravenous administration in crossbred calves," Veterinary Research Communications, vol. 31, no. 7, pp. 873-879, 2007.

[21] A. Goudah and K. Abo-El-Sooud, "Pharmacokinetics, urinary excretion and milk penetration of levofloxacin in lactating 
goats," Journal of Veterinary Pharmacology and Therapeutics, vol. 32, no. 1, pp. 101-104, 2009.

[22] G. A. Albarellos, L. A. Ambros, and M. F. Landoni, "Pharmacokinetics of levofloxacin after single intravenous and repeat oral administration to cats," Journal of Veterinary Pharmacology and Therapeutics, vol. 28, no. 4, pp. 363-369, 2005.

[23] A. Goudah, "Pharmacokinetics of levofloxacin in male camels (Camelus dromedarius)," Journal of Veterinary Pharmacology and Therapeutics, vol. 32, no. 3, pp. 296-299, 2009.

[24] A. Goudah, K. Abo El-Sooud, J.-H. Shim, H.-C. Shin, and A. M. Abd El-Aty, "Characterization of the pharmacokinetic disposition of levofloxacin in stallions after intravenous and intramuscular administration," Journal of Veterinary Pharmacology and Therapeutics, vol. 31, no. 5, pp. 399-405, 2008.

[25] M. I. R. M. Santoro, N. M. Kassab, A. K. Singh, and E. R. M. Kedor-Hackmam, "Quantitative determination of gatifloxacin, levofloxacin, lomefloxacin and pefloxacin fluoroquinolonic antibiotics in pharmaceutical preparations by high-performance liquid chromatography," Journal of Pharmaceutical and Biomedical Analysis, vol. 40, no. 1, pp. 179-184, 2006.

[26] A. W. Craig and B. Suh, "Protein binding and the antibacterial effects. Method for the determination of protein binding," in Antibiotics in laboratory Medicine, V. Lorian, Ed., pp. 367-402, Williams \& Wilkins, Baltimore, Md, USA, 3rd edition, 1991.

[27] K. Yamaoka, T. Nakagawa, and T. Uno, "Statistical moments in pharmacokinetics," Journal of Pharmacokinetics and Biopharmaceutics, vol. 6, no. 6, pp. 547-558, 1978.

[28] M. Gibaldi and D. Perrier, Pharmacokinetics, Marcel Dekker, New York, NY, USA, 2nd edition, 1982.

[29] S. A. Marshall and R. N. Jones, "In vitro activity of DU-6859a, a new fluorocyclopropyl quinolone," Antimicrobial Agents and Chemotherapy, vol. 37, no. 12, pp. 2747-2753, 1993.

[30] P. L. Toutain and A. Bousquet-Mélou, "Volumes of distribution," Journal of Veterinary Pharmacology and Therapeutics, vol. 27, no. 6, pp. 441-453, 2004.

[31] S. A. Brown, "Fluoroquinolones in animal health," Journal of Veterinary Pharmacology and Therapeutics, vol. 19, no. 1, pp. 1-14, 1996.

[32] E. Fernández-Varón, L. Villamayor, E. Escudero, A. Espuny, and C. M. Cárceles, "Pharmacokinetics and milk penetration of moxifloxacin after intravenous and subcutaneous administration to lactating goats," Veterinary Journal, vol. 172, no. 2, pp. 302-307, 2006.

[33] J.-L. Riond, K. Tyczkowska, and J. E. Riviere, "Pharmacokinetics and metabolic inertness of doxycycline in calves with mature or immature rumen function," American Journal of Veterinary Research, vol. 50, no. 8, pp. 1329-1333, 1989.

[34] A. Goudah, "Disposition kinetics of moxifloxacin in lactating ewes," Veterinary Journal, vol. 178, no. 2, pp. 282-287, 2008.

[35] J. Turnidge, "Pharmacokinetics and pharmacodynamics of fluoroquinolones,” Drugs, vol. 58, no. 2, pp. 29-36, 1999.

[36] G. Zlotos, A. Bücker, M. Kinzig-Schippers, F. Sorgel, and U. Holzgrabe, "Plasma protein binding of gyrase inhibitors," Journal of Pharmaceutical Sciences, vol. 87, no. 2, pp. 215-220, 1998.

[37] H. Lode, K. Borner, and P. Koeppe, "Pharmacodynamics of fluoroquinolones," Clinical Infectious Diseases, vol. 27, no. 1, pp. 33-39, 1998.
[38] P. L. Toutain, J. R. E. Del Castillo, and A. BousquetMélou, "The pharmacokinetic-pharmacodynamic approach to a rational dosage regimen for antibiotics," Research in Veterinary Science, vol. 73, no. 2, pp. 105-114, 2002.

[39] R. D. Walker, "The use of fluoroquinolones for companion animal antimicrobial therapy," Australian Veterinary Journal, vol. 78, no. 2, pp. 84-90, 2000. 

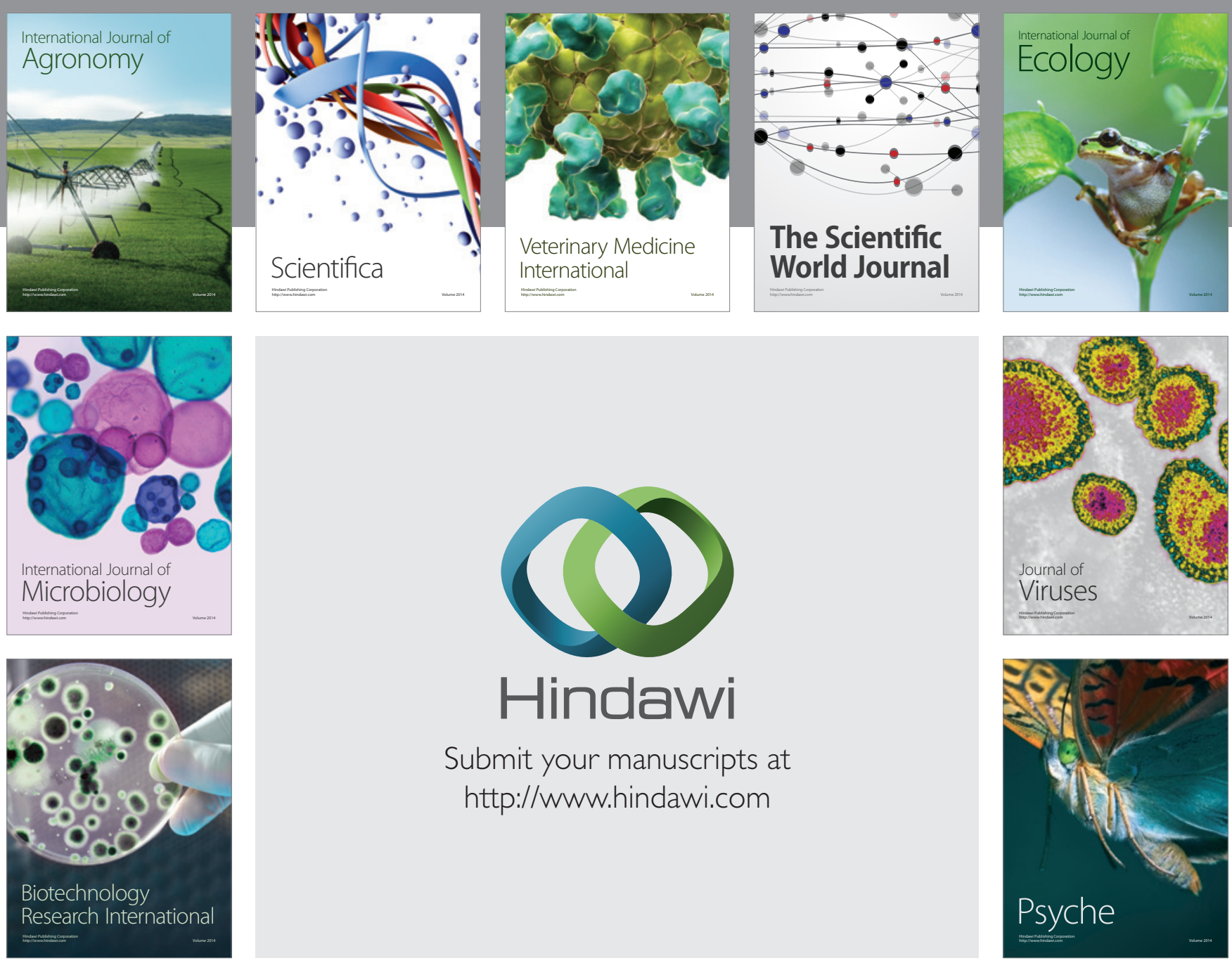

Submit your manuscripts at

http://www.hindawi.com
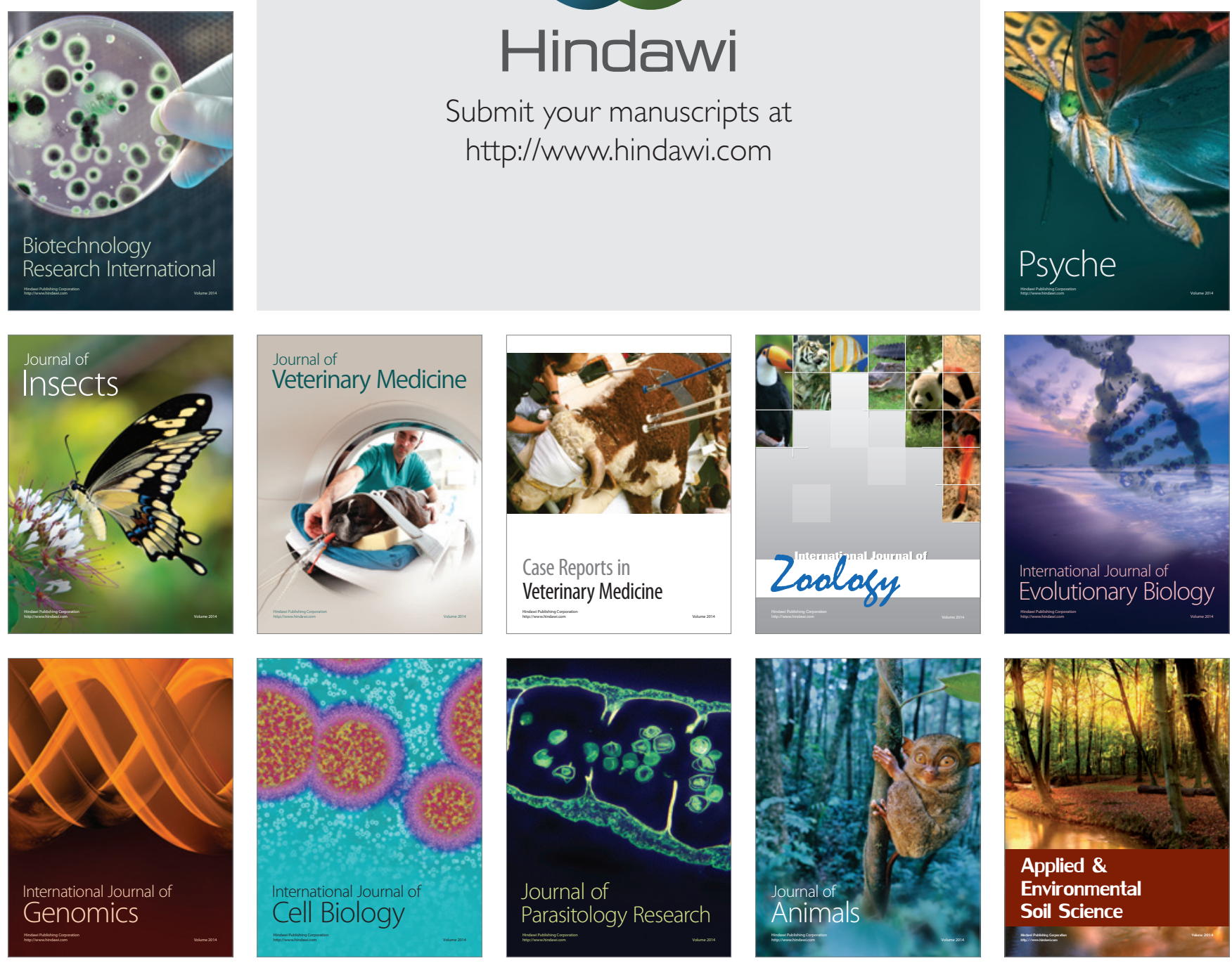